\title{
Precision Measurement of the Helium Flux in Primary Cosmic Rays of Rigidities 1.9 GV to 3 TV with the Alpha Magnetic Spectrometer on the International Space Station
}

M. Aguilar ${ }^{26}$ D. Aisa,${ }^{32,33}$ B. Alpat, ${ }^{32}$ A. Alvino, ${ }^{32}$ G. Ambrosi, ${ }^{32}$ K. Andeen, ${ }^{22}$ L. Arruda, ${ }^{24}$ N. Attig, ${ }^{21}$ P. Azzarello, ${ }^{16}$ A. Bachlechner, ${ }^{1}$ F. Barao, ${ }^{24}$ A. Barrau, ${ }^{17}$ L. Barrin, ${ }^{15}$ A. Bartoloni, ${ }^{38}$ L. Basara, ${ }^{36}$ M. Battarbee,${ }^{47}$ R. Battiston,${ }^{36,37, a}$ J. Bazo, ${ }^{32, b}$ U. Becker, ${ }^{9}$ M. Behlmann, ${ }^{9}$ B. Beischer, ${ }^{1}$ J. Berdugo, ${ }^{26}$ B. Bertucci, ${ }^{32,33}$ V. Bindi, ${ }^{19}$ S. Bizzaglia, ${ }^{32}$ M. Bizzarri, ${ }^{32,33}$ G. Boella, ${ }^{28,29}$ W. de Boer, ${ }^{22}$ K. Bollweg, ${ }^{20}$ V. Bonnivard, ${ }^{17}$ B. Borgia, ${ }^{38,39}$ S. Borsini,${ }^{32}$ M. J. Boschini, ${ }^{28}$ M. Bourquin, ${ }^{16}$ J. Burger, ${ }^{9}$ F. Cadoux,${ }^{16}$ X. D. Cai, ${ }^{9}$ M. Capell, ${ }^{9}$ S. Caroff, ${ }^{3}$ J. Casaus, ${ }^{26}$ G. Castellini, ${ }^{14}$ I. Cernuda, ${ }^{26}$ D. Cerreta, ${ }^{32,33}$ F. Cervelli, ${ }^{34}$ M. J. Chae, ${ }^{41}$ Y. H. Chang, ${ }^{10}$ A. I. Chen, ${ }^{9}$ G. M. Chen, ${ }^{6}$ H. Chen, ${ }^{9}$ H. S. Chen, ${ }^{6}$ L. Cheng, ${ }^{42}$ H. Y. Chou, ${ }^{10}$ E. Choumilov, ${ }^{9}$ V. Choutko, ${ }^{9}$ C. H. Chung, ${ }^{1}$ C. Clark, ${ }^{20}$ R. Clavero, ${ }^{23}$ G. Coignet, ${ }^{3}$ C. Consolandi, ${ }^{19}$ A. Contin, ${ }^{7,8}$ C. Corti, ${ }^{19}$ E. Cortina Gil,${ }^{16, c}$ B. Coste,${ }^{36,15}$ W. Creus, ${ }^{10}$ M. Crispoltoni, ${ }^{32,33}$ Z. Cui, ${ }^{42}$ Y. M. Dai, ${ }^{5}$ C. Delgado, ${ }^{26}$ S. Della Torre ${ }^{28}$ M. B. Demirköz, ${ }^{2}$ L. Derome, ${ }^{17}$ S. Di Falco,${ }^{34}$ L. Di Masso, ${ }^{32,33}$ F. Dimiccoli, ${ }^{36,37}$ C. Díaz, ${ }^{26}$ P. von Doetinchem, ${ }^{19}$ F. Donnini, ${ }^{32,33}$ M. Duranti, ${ }^{32,33}$ D. D'Urso, ${ }^{32, d}$ A. Egorov, ${ }^{9}$ A. Eline, ${ }^{9}$ F. J. Eppling,,${ }^{9}$ T. Eronen, ${ }^{47}$ Y. Y. Fan, ${ }^{46, \mathrm{e}}$ L. Farnesini, ${ }^{32}$ J. Feng, ${ }^{3,46, \mathrm{f}}$ E. Fiandrini, ${ }^{32,33}$ A. Fiasson, ${ }^{3}$ E. Finch, ${ }^{31}$ P. Fisher, ${ }^{9}$ V. Formato, ${ }^{32,15}$ Y. Galaktionov, ${ }^{9}$ G. Gallucci, ${ }^{34}$ B. García, ${ }^{26}$ R. García-López, ${ }^{23}$ C. Gargiulo, ${ }^{15}$ H. Gast, ${ }^{1}$ I. Gebauer ${ }^{22}$ M. Gervasi, ${ }^{28,29}$ A. Ghelfi, ${ }^{17}$ F. Giovacchini,${ }^{26}$ P. Goglov, ${ }^{9}$ J. Gong, ${ }^{30}$ C. Goy, ${ }^{3}$ V. Grabski ${ }^{27}$ D. Grandi ${ }^{28}$ M. Graziani,${ }^{32,33}$ C. Guandalini, ${ }^{7}$ I. Guerri, ${ }^{34,35}$ K. H. Guo, ${ }^{18}$ D. Haas, ${ }^{16,9}$ M. Habiby, ${ }^{16}$ S. Haino, ${ }^{46}$ K. C. Han, ${ }^{25}$ Z. H. He, ${ }^{18}$ M. Heil,${ }^{9}$ J. Hoffman, ${ }^{10,19}$ T. H. Hsieh, ${ }^{9}$ Z. C. Huang, ${ }^{18}$ C. Huh,${ }^{13}$ M. Incagli, ${ }^{34}$ M. Ionica, ${ }^{32}$ W. Y. Jang, ${ }^{13}$ H. Jinchi, ${ }^{25}$ K. Kanishev, ${ }^{36,37,15}$ G. N. Kim, ${ }^{13}$ K. S. Kim, ${ }^{13}$ Th. Kirn, ${ }^{1}$ M. A. Korkmaz, ${ }^{2}$ R. Kossakowski, ${ }^{3}$ O. Kounina, ${ }^{9}$ A. Kounine, ${ }^{9}$ V. Koutsenko, ${ }^{9}$ M. S. Krafczyk, ${ }^{9}$ G. La Vacca, ${ }^{28}$ E. Laudi, ${ }^{32,33, h}$ G. Laurenti, ${ }^{7}$ I. Lazzizzera, ${ }^{36,37}$ A. Lebedev, ${ }^{9}$ H. T. Lee, ${ }^{45}$ S. C. Lee, ${ }^{46}$ C. Leluc, ${ }^{16}$ H. L. Li, ${ }^{46, i}$ J. Q. Li, ${ }^{9, j}$ J. Q. Li ${ }^{30}$ Q. Li, ${ }^{30}$ Q. Li, ${ }^{9, j}$ T. X. Li, ${ }^{18}$ W. Li, ${ }^{4}$ Y. Li, ${ }^{16, f}$ Z. H. Li, ${ }^{6}$ Z. Y. Li, ${ }^{46, f}$ S. Lim, ${ }^{13}$ C. H. Lin, ${ }^{46}$ P. Lipari, ${ }^{38}$ T. Lippert, ${ }^{21}$ D. Liu, ${ }^{46}$ H. Liu, ${ }^{30}$ Hu Liu, ${ }^{26, k}$ M. Lolli, ${ }^{7}$ T. Lomtadze,${ }^{34}$ M. J. Lu, ${ }^{36, *}{ }^{*}$ S. Q. Lu, ${ }^{46, f}$ Y. S. Lu, ${ }^{6}$ K. Luebelsmeyer, ${ }^{1}$ F. Luo, ${ }^{42}$ J.Z. Luo, ${ }^{30}$ S. S. Lv, ${ }^{18}$ R. Majka, ${ }^{31}$ C. Mañá, ${ }^{26}$ J. Marín, ${ }^{26}$ T. Martin, ${ }^{20}$ G. Martínez,${ }^{26}$ N. Masi, ${ }^{7}$ D. Maurin,${ }^{17}$ A. Menchaca-Rocha, ${ }^{27}$ Q. Meng, ${ }^{30}$ D. C. Mo, ${ }^{18}$ L. Morescalchi, ${ }^{34, m}$ P. Mott, ${ }^{20}$ M. Müller, ${ }^{1}$ T. Nelson, ${ }^{19}$ J. Q. Ni, ${ }^{18}$ N. Nikonov, ${ }^{22}$ F. Nozzoli, ${ }^{32, d}$ P. Nunes, ${ }^{24}$ A. Obermeier, ${ }^{1}$ A. Oliva, ${ }^{26}$ M. Orcinha ${ }^{24}$ F. Palmonari, ${ }^{7,8}$ C. Palomares, ${ }^{26}$ M. Paniccia ${ }^{16}$ A. Papi, ${ }^{32}$ M. Pauluzzi, ${ }^{32,33}$ E. Pedreschi, ${ }^{34}$ S. Pensotti, ${ }^{28,29}$ R. Pereira, ${ }^{19}$ N. Picot-Clemente, ${ }^{12}$ F. Pilo, ${ }^{34}$ A. Piluso, ${ }^{32,33}$ C. Pizzolotto, ${ }^{32, \mathrm{~d}}$ V. Plyaskin, ${ }^{9}$ M. Pohl,${ }^{16}$ V. Poireau, ${ }^{3}$ A. Putze, ${ }^{3, n}$ L. Quadrani,${ }^{7,8}$ X. M. Qi ${ }^{18}$ X. Qin, ${ }^{32, i}$ Z. Y. Qu ${ }^{46,0}$ T. Räihä, ${ }^{1}$ P. G. Rancoita, ${ }^{28}$ D. Rapin,${ }^{16}$ J. S. Ricol, ${ }^{17}$ I. Rodríguez, ${ }^{26}$ S. Rosier-Lees,${ }^{3}$ A. Rozhkov, ${ }^{9}$ D. Rozza, ${ }^{28}$

R. Sagdeev, ${ }^{11}$ J. Sandweiss, ${ }^{31}$ P. Saouter, ${ }^{16}$ S. Schael, ${ }^{1}$ S. M. Schmidt, ${ }^{21}$ A. Schulz von Dratzig, ${ }^{1}$ G. Schwering, ${ }^{1}$ G. Scolieri ${ }^{32}$ E. S. Seo, ${ }^{12}$ B. S. Shan, ${ }^{4}$ Y. H. Shan, ${ }^{4}$ J. Y. Shi, ${ }^{30}$ X. Y. Shi, ${ }^{9, p}$ Y. M. Shi, ${ }^{43}$ T. Siedenburg, ${ }^{1}$ D. Son, ${ }^{13}$ J. W. Song, ${ }^{42}$ F. Spada ${ }^{38}$ F. Spinella, ${ }^{34}$ W. Sun, ${ }^{9}$ W. H. Sun,,${ }^{9,}$ M. Tacconi, ${ }^{28,15}$ C. P. Tang, ${ }^{18}$ X. W. Tang, ${ }^{6}$ Z. C. Tang, ${ }^{6}$ L. Tao, ${ }^{3}$ D. Tescaro, ${ }^{23}$ Samuel C. C. Ting, ${ }^{9}$ S. M. Ting, ${ }^{9}$ N. Tomassetti,${ }^{17}$ J. Torsti ${ }^{47}$ C. Türkoğlu, ${ }^{2}$ T. Urban, ${ }^{20}$ V. Vagelli, ${ }^{22,32}$ E. Valente, ${ }^{38,39}$ C. Vannini, ${ }^{34}$ E. Valtonen, ${ }^{47}$ S. Vaurynovich, ${ }^{9}$ M. Vecchi,${ }^{40}$ M. Velasco, ${ }^{26}$ J. P. Vialle, ${ }^{3}$ V. Vitale, ${ }^{32, d}$ S. Vitillo, ${ }^{16}$ L. Q. Wang, ${ }^{42}$ N. H. Wang, ${ }^{42}$ Q. L. Wang, ${ }^{5}$ R. S. Wang, ${ }^{43}$ X. Wang, ${ }^{9}$ Z. X. Wang, ${ }^{18}$ Z. L. Weng, ${ }^{9}$ K. Whitman, ${ }^{19}$ J. Wienkenhöver, ${ }^{1}$ M. Willenbrock, ${ }^{9}$ H. Wu ${ }^{30}$ X. Wu, ${ }^{16}$ X. Xia, ${ }^{26, i}$ M. Xie, ${ }^{9, j}$ S. Xie, ${ }^{43}$ R. Q. Xiong, ${ }^{30}$ N. S. Xu, ${ }^{18}$ W. Xu,${ }^{9}$ Q. Yan, ${ }^{9}$ J. Yang, ${ }^{41}$ M. Yang, ${ }^{6}$ Y. Yang, ${ }^{44}$ Q. H. Ye, ${ }^{43}$ H. Yi,${ }^{30}$ Y. J. Yu, Z. Q. Yu, ${ }^{6}$ S. Zeissler, ${ }^{22}$ C. Zhang, ${ }^{6}$ J. H. Zhang, ${ }^{30}$ M. T. Zhang, ${ }^{18}$ S. D. Zhang, ${ }^{9, j}$ S. W. Zhang, ${ }^{6}$ X. B. Zhang, ${ }^{18}$ Z. Zhang, ${ }^{18}$ Z. M. Zheng, ${ }^{4}$ H. L. Zhuang, ${ }^{6}$ V. Zhukov, ${ }^{1}$ A. Zichichi, ${ }^{7,8}$ N. Zimmermann, ${ }^{1}$ and P. Zuccon ${ }^{9}$

(AMS Collaboration)

\author{
${ }^{1}$ I. Physics Institute and JARA-FAME, RWTH Aachen University, D-52056 Aachen, Germany \\ ${ }^{2}$ Department of Physics, Middle East Technical University (METU), 06800 Ankara, Turkey \\ ${ }^{3}$ Laboratoire d'Annecy-le-Vieux de Physique des Particules (LAPP), CNRS/IN2P3 and Université de Savoie Mont Blanc, \\ F-74941 Annecy-le-Vieux, France \\ ${ }^{4}$ Beihang University (BUAA), Beijing 100191, China \\ ${ }^{5}$ Institute of Electrical Engineering (IEE), Chinese Academy of Sciences, Beijing 100190, China \\ ${ }^{6}$ Institute of High Energy Physics (IHEP), Chinese Academy of Sciences, Beijing 100039, China \\ ${ }^{7}$ INFN Sezione di Bologna, I-40126 Bologna, Italy \\ ${ }^{8}$ Università di Bologna, I-40126 Bologna, Italy \\ ${ }^{9}$ Massachusetts Institute of Technology (MIT), Cambridge, Massachusetts 02139, USA \\ ${ }^{10}$ National Central University (NCU), Chung-Li, Tao Yuan 32054, Taiwan
}


${ }^{11}$ East-West Center for Space Science, University of Maryland, College Park, Maryland 20742, USA

${ }^{12}$ IPST, University of Maryland, College Park, Maryland 20742, USA

${ }^{13}$ CHEP, Kyungpook National University, 702-701 Daegu, Korea

${ }^{14}$ CNR-IROE, I-50125 Firenze, Italy

${ }^{15}$ European Organization for Nuclear Research (CERN), CH-1211 Geneva 23, Switzerland

${ }^{16}$ DPNC, Université de Genève, CH-1211 Genève 4, Switzerland

${ }^{17}$ Laboratoire de Physique Subatomique et de Cosmologie (LPSC), CNRS/IN2P3 and Université Grenoble-Alpes, F-38026 Grenoble, France

${ }^{18}$ Sun Yat-Sen University (SYSU), Guangzhou 510275, China

${ }^{19}$ Physics and Astronomy Department, University of Hawaii, Honolulu, Hawaii 96822, USA

${ }^{20}$ National Aeronautics and Space Administration Johnson Space Center (JSC), and Jacobs-Sverdrup, Houston, Texas 77058, USA

${ }^{21}$ Jülich Supercomputing Centre and JARA-FAME, Research Centre Jülich, D-52425 Jülich, Germany

${ }^{22}$ Institut für Experimentelle Kernphysik, Karlsruhe Institute of Technology (KIT), D-76128 Karlsruhe, Germany

${ }^{23}$ Instituto de Astrofísica de Canarias (IAC), E-38205 La Laguna, and Departamento de Astrofísica, Universidad de La Laguna, E-38206 La Laguna, Tenerife, Spain

${ }^{24}$ Laboratório de Instrumentação e Física Experimental de Partículas (LIP), P-1000 Lisboa, Portugal

${ }^{25}$ National Chung-Shan Institute of Science and Technology (NCSIST), Longtan, Tao Yuan 325, Taiwan

${ }^{26}$ Centro de Investigaciones Energéticas, Medioambientales y Tecnológicas (CIEMAT), E-28040 Madrid, Spain

${ }^{27}$ Instituto de Física, Universidad Nacional Autónoma de México (UNAM), México, D. F. 01000 Mexico

${ }^{28}$ INFN Sezione di Milano-Bicocca, I-20126 Milano, Italy

${ }^{29}$ Università di Milano-Bicocca, I-20126 Milano, Italy

${ }^{30}$ Southeast University (SEU), Nanjing 210096, China

${ }^{31}$ Physics Department, Yale University, New Haven, Connecticut 06520, USA

${ }^{32}$ INFN Sezione di Perugia, I-06100 Perugia, Italy

${ }^{33}$ Università di Perugia, I-06100 Perugia, Italy

${ }^{34}$ INFN Sezione di Pisa, I-56100 Pisa, Italy

${ }^{35}$ Università di Pisa, I-56100 Pisa, Italy

${ }^{36}$ INFN TIFPA, I-38123 Povo, Trento, Italy

${ }^{37}$ Università di Trento, I-38123 Povo, Trento, Italy

${ }^{38}$ INFN Sezione di Roma 1, I-00185 Roma, Italy

${ }^{39}$ Università di Roma La Sapienza, I-00185 Roma, Italy

${ }^{40}$ Instituto de Fìsica de São Carlos, Universidade de São Paulo, CP 369, 13560-970 São Carlos, São Paulo, Brazil

${ }^{41}$ Department of Physics, Ewha Womans University, Seoul 120-750, Korea

${ }^{42}$ Shandong University (SDU), Jinan, Shandong 250100, China

${ }^{43}$ Shanghai Jiaotong University (SJTU), Shanghai 200030, China

${ }^{44}$ National Cheng Kung University, Tainan 701, Taiwan

${ }^{45}$ Academia Sinica Grid Center (ASGC), Nankang, Taipei 11529, Taiwan

${ }^{46}$ Institute of Physics, Academia Sinica, Nankang, Taipei 11529, Taiwan

${ }^{47}$ Space Research Laboratory, Department of Physics and Astronomy, University of Turku, FI-20014 Turku, Finland

(Received 25 August 2015; published 17 November 2015)

Knowledge of the precise rigidity dependence of the helium flux is important in understanding the origin, acceleration, and propagation of cosmic rays. A precise measurement of the helium flux in primary cosmic rays with rigidity (momentum/charge) from $1.9 \mathrm{GV}$ to $3 \mathrm{TV}$ based on 50 million events is presented and compared to the proton flux. The detailed variation with rigidity of the helium flux spectral index is presented for the first time. The spectral index progressively hardens at rigidities larger than $100 \mathrm{GV}$. The rigidity dependence of the helium flux spectral index is similar to that of the proton spectral index though the magnitudes are different. Remarkably, the spectral index of the proton to helium flux ratio increases with rigidity up to $45 \mathrm{GV}$ and then becomes constant; the flux ratio above $45 \mathrm{GV}$ is well described by a single power law.

DOI: 10.1103/PhysRevLett.115.211101

Published by the American Physical Society under the terms of the Creative Commons Attribution 3.0 License. Further distribution of this work must maintain attribution to the author(s) and the published article's title, journal citation, and DOI.
PACS numbers: 98.70.Sa, 95.55.Vj, 95.85.Ry, 96.50.sb

Helium nuclei in cosmic rays are believed to be mainly produced by Galactic sources such as supernova remnants. Precise knowledge of the helium $(\mathrm{He})$ spectrum in the GV-TV rigidity region gives important information on the origin, acceleration, and subsequent propagation processes of cosmic rays in the Galaxy [1]. 
Recent important measurements of the He flux in cosmic rays have reported different variations of the flux with energy [2-6]. In particular, the ATIC-2, CREAM, and PAMELA experiments showed deviations of the helium flux from a single power law. Many models based on different sources, acceleration mechanisms, and diffusive propagation effects [7] were proposed to account for the hardening of the He flux as well as for the difference of the proton and He fluxes. In this Letter we report the precise measurement of the helium flux in primary cosmic rays in the rigidity range from $1.9 \mathrm{GV}$ to $3 \mathrm{TV}$ based on data collected by the Alpha Magnetic Spectrometer (AMS) during the first 30 months (May 19, 2011 to November 26, 2013) of operation onboard the International Space Station (ISS). Using our measurement of the proton flux with the same data set [8], this Letter also reports the characteristics of the ratio of the proton flux to the helium flux.

Detector.-The AMS is a general purpose high energy magnetic spectrometer in space. The layout and description of the detector are presented in Ref. [9]. The key elements used in this measurement are the permanent magnet [10], the silicon tracker, four planes of time of flight (TOF) scintillation counters, and an array of 16 anticoincidence counters (ACC). The AMS also contains a transition radiation detector (TRD), a ring imaging Čerenkov detector (RICH), and an electromagnetic calorimeter (ECAL).

The AMS coordinate system is concentric with the magnet and above, below, and downward-going refer to the AMS coordinate system. Timing, location, and orientation are provided by GPS units affixed to the AMS and to the ISS. The detector performance has been steady over time.

The tracker [11] has nine layers, the first (L1) at the top of the detector, the second (L2) above the magnet, six (L3 to L8) within the bore of the magnet, and the last (L9) above the ECAL. L2 to L8 constitute the inner tracker. The tracker accurately determines the trajectory of cosmic rays by multiple measurements of the coordinates. Together, the tracker and the magnet measure the rigidity $R$ of charged cosmic rays. For $Z=2$ particles, the spatial resolution in each tracker layer is $7.5 \mu \mathrm{m}$ in the bending direction and the maximum detectable rigidity (MDR) is $3.2 \mathrm{TV}$ over the $3 \mathrm{~m}$ lever arm from L1 to L9.

Each layer of the tracker also provides an independent measurement of the charge $Z$ of the cosmic ray. The charge resolution of the combined inner tracker is $\Delta Z \simeq 0.07$ for $Z=2$ particles.

Two planes of TOF counters [12] are located above L2 and two planes are located below the magnet. The overall velocity $(\beta=v / c)$ resolution has been measured to be $\Delta \beta / \beta^{2}=0.02$ for $Z=2$ particles. This discriminates between upward- and downward-going particles. The pulse heights of the two upper layers are combined to provide an independent measurement of the charge with an accuracy $\Delta Z \simeq 0.08$ for $Z=2$. The pulse heights from the two lower planes are combined to provide another independent charge measurement with the same accuracy.

The 16 anticoincidence counters (ACC) form a cylindrical shell between the inner tracker and the magnet. Adjacent counters are combined to provide 8 readout sectors. The ACC have an efficiency of 0.99999 to reject cosmic rays which enter the inner tracker from the side.

Helium traversing the AMS were triggered and flagged by the logical OR of any of three trigger conditions onboard the ISS: (i) the coincidence, within $240 \mathrm{ns,} \mathrm{of} \mathrm{signals} \mathrm{from}$ all four TOF planes each with a pulse height above 0.5 of a minimum ionizing particle signal (MIP, $Z=1$ ) together with an absence of signals from the ACC; OR (ii) the coincidence, within $240 \mathrm{~ns}$, of signals from all four TOF planes each with pulse heights above 3.5 times a MIP signal together with signals from no more than 4 out of the $8 \mathrm{ACC}$ sectors; OR (iii) the coincidence, within $240 \mathrm{~ns}$, of 3 out of the 4 TOF layers each with pulse heights above 0.5 of a MIP signal and with no ACC requirement. Condition (iii) was prescaled to $1 \%$; i.e., only 1 event out of 100 which met these conditions was passed on to the OR. The efficiency of trigger (iii) was estimated directly from the data to be above $99.99 \%$ for all rigidities using $Z=2$ events in which 1 of the 4 TOF layers gave no signal. This allowed the estimation of the efficiency of each TOF layer and, consequently, the efficiency of trigger (iii). Trigger (iii) is used to measure the efficiency of triggers (i) and (ii). Together, triggers (i) and (ii) ensure a high efficiency (see the Analysis section) of detecting cosmic ray ions while effectively rejecting cosmic ray events entering the inner tracker from the side.

Monte Carlo simulated events were produced using a dedicated program developed by the collaboration based on the GEANT-4.10.1 package [13]. The program simulates electromagnetic and hadronic interactions of particles in the material of the AMS and generates detector responses. The INCL ++ package [14] was used to model heliumnuclear inelastic interactions below $5 \mathrm{GeV} /$ nucleon and the DPMJET-II.5 package [15] was used at higher energies. The helium-nuclear elastic and quasielastic scattering were modeled using the measurements from Ref. [16]. As will be discussed below, we have developed a method to measure the helium-nuclear interactions with the materials in the AMS and used this measurement to scale the model predictions. The digitization of signals is simulated precisely according to the measured characteristics of the electronics. The simulated events then undergo the same reconstruction as used for the data.

Selection. - In the first 30 months $\left(7.96 \times 10^{7} \mathrm{~s}\right)$ the AMS collected $4.1 \times 10^{10}$ cosmic ray events. The collection time used in this analysis includes only those seconds during which the detector was in normal operating conditions and, in addition, the AMS was pointing within $40^{\circ}$ of the local zenith, the data acquisition live time exceeded $50 \%$ (compared to its typical value of $90 \%$ ), and the ISS 
was outside of the South Atlantic Anomaly. Because of the influence of the geomagnetic field, this collection time for primary cosmic rays increases with increasing rigidity becoming constant at $6.29 \times 10^{7} \mathrm{~s}$ above $30 \mathrm{GV}$ [8].

By selecting events to be downward going and to have a reconstructed track in the inner tracker with charge compatible with $Z=2$, i.e., $1.7<Z_{\mathrm{L} 2 \text { to } \mathrm{L} 8}<2.5$, we obtain $1.7 \times 10^{9}$ events. In order to have the best resolution at the highest rigidities, further selections are made by requiring the track to pass through L1 and L9 and to satisfy additional track fitting quality criteria such as a $\chi^{2} /$ d.f. $<10$ in the bending coordinate. To remove the helium candidates which interacted within the detector, the charge as measured by each of L1, the upper TOF, the lower TOF, and $L 9$ is required to be compatible with $Z=2$, namely, $1.6<Z_{\mathrm{L} 1}<2.9,1.25<Z_{\text {upper TOF }}, 1.25<Z_{\text {lower TOF }}$, and $1.6<Z_{\mathrm{L} 9}<2.9$. To select only primary cosmic rays, the measured rigidity is required to be greater than a factor of 1.2 times the maximum geomagnetic cutoff within the AMS field of view. The cutoff was calculated by backtracing [17] particles from the top of the AMS out to 50 Earth's radii using the most recent IGRF [18] geomagnetic model. These procedures resulted in a sample of $5.0 \times 10^{7}$ primary cosmic rays with $Z=2$, where all the events passed from L1 to L9.

Because of the multiple independent measurements of the charge, the selected sample contains only a small contamination of particles which had $Z \neq 2$ at the top of the AMS. Comparing the proton and helium charge distributions in the inner tracker, the proton contamination of the helium sample was measured to be less than $10^{-4}$ over the entire rigidity range. The sample also contains helium from other nuclei which interact at the top of the AMS (for example, in L1). From the measured flux [19] and Monte Carlo simulation, this contribution is below $10^{-3}$ for the entire rigidity range. The background contributions are subtracted from the flux and the uncertainties are accounted for in the systematic errors.

Analysis. - The isotropic He flux $\Phi_{i}$ for the $i$ th rigidity bin $\left(R_{i}, R_{i}+\Delta R_{i}\right)$ is

$$
\Phi_{i}=\frac{N_{i}}{A_{i} \epsilon_{i} T_{i} \Delta R_{i}},
$$

where $N_{i}$ is the number of events corrected with the rigidity resolution function (see below), $A_{i}$ is the effective acceptance, $\epsilon_{i}$ is the trigger efficiency, and $T_{i}$ is the collection time. In this Letter the helium flux was measured in 68 bins, $i=1$ to 68 , from $1.9 \mathrm{GV}$ to $3 \mathrm{TV}$ with bin widths chosen according to the rigidity resolution and to the proton flux measurement [8]. The trigger efficiency $\epsilon_{i}$ was measured to range from $95 \%$ to $99.5 \%$, where the inefficiency is mostly due to secondary $\delta$ rays produced by $\mathrm{He}$ in the tracker materials and which then entered the ACC. The Monte Carlo simulation agrees with the measured trigger efficiency within $0.5 \%$.
The bin-to-bin migration of events was corrected using the two unfolding procedures described in Ref. [8]. This correction, $\left(N_{i}-\boldsymbol{\aleph}_{i}\right) / \boldsymbol{\aleph}_{i}$, where $\boldsymbol{\aleph}_{i}$ is the number of observed events in bin $i$, is $+15 \%$ at $2 \mathrm{GV},+5 \%$ at $3 \mathrm{GV}$ decreasing smoothly to $-5 \%$ at $300 \mathrm{GV}$, and reaches $-19 \%$ at $3 \mathrm{TV}$.

Extensive studies were made of the systematic errors. These errors include the uncertainties in the trigger efficiency, the geomagnetic cutoff factor, the acceptance taking into account the event selection and reconstruction and also accounting for helium interactions in the detector, the unfolding, the rigidity resolution function, the absolute rigidity scale, and the negligible background contamination discussed above. The trigger efficiency error is dominated by the statistics available from the $1 \%$ prescaled trigger (iii) event sample. It is less than $0.2 \%$ below $100 \mathrm{GV}$ and reaches $1 \%$ at $3 \mathrm{TV}$. The geomagnetic cutoff factor was varied from 1.0 to 1.4 , resulting in a negligible systematic uncertainty (less than $0.1 \%$ ) in the whole rigidity range.

The effective acceptance $A_{i}$ was calculated from the Monte Carlo simulation and then corrected for small differences between the data and the Monte Carlo samples related to the event reconstruction and selection, namely, in the efficiencies of velocity determination, track finding, charge determination, and tracker quality cuts. Together, the correction on the acceptance was found to be less than $2 \%$ above $2 \mathrm{GV}$. The corresponding systematic error on the flux is less than $1 \%$ below $200 \mathrm{GV}$ and reaches $1.4 \%$ at $3 \mathrm{TV}$.

Averaged over path lengths within the acceptance, the material traversed by particles between L1 and L9 is composed, by weight, of $73 \%$ carbon, $17 \%$ aluminum, and small amounts of silicon, oxygen, hydrogen, sodium, gold, and other elements. The corresponding inelastic cross sections of $\mathrm{He}+\mathrm{C}$ and $\mathrm{He}+\mathrm{Al}$ have only been measured below $10 \mathrm{GV}$ [20]. To accurately determine the effect on the acceptance of He interactions in the detector, we have developed a method [21] to determine the magnitude and rigidity dependence of the survival probability of $\mathrm{He}$ when traversing the detector materials. We used a sample of primary cosmic rays collected with the AMS horizontal, that is, when the ISS was oriented such that the AMS was pointing within $90^{\circ} \pm 10^{\circ}$ of the local zenith, a total of $1.4 \times 10^{5} \mathrm{~s}$. In this condition, primary helium can travel from L9 to L1 and from L1 to L9. Particles which passed through from L8 to L2 were identified as $Z=2$ with the $d E / d x$ in the seven inner tracker layers L8 to L2. We then measured the survival probability for helium to traverse the material from L2 to L1 (the upper TOF and TRD) by comparing the charge distributions between L2 and L1.

The small $(<3 \%)$ interaction probability between tracker L2 and L8 was calculated by comparing the charge distributions between the upper and the lower TOF for He events.

Using particles collected with the AMS horizontal, which passed from L2 to L8 and were identified as $Z=2$, the He survival probability was also measured 
between L8 and L9, that is, when traversing the thin aluminum and carbon fiber inner tracker enclosure, the lower TOF, and the RICH radiator. The accuracy of this method was verified using the data collected when the AMS was in normal data taking conditions.

The Glauber-Gribov model [13] of inelastic cross sections is used in the Monte Carlo calculation of the acceptance. In this model the corresponding cross sections have small rigidity dependencies of $5 \%$ from $8 \mathrm{GV}$ to $3 \mathrm{TV}$. To obtain the best agreement between data and simulation for the He survival probabilities measured using the method described above, dedicated event samples were simulated with the inelastic cross sections scaled up by $1.05,1.10$, 1.15, and 1.20. Then the survival probabilities for $\mathrm{He}$ between L2 and L1 and between L8 and L9 were compared between data and the dedicated simulated event samples. The interpolated scaling with the best agreement to data above $30 \mathrm{GV}$ was chosen. Figure SM1(a) in Ref. [22] shows the ratio between the simulation and data of the $\mathrm{He}$ survival probabilities from L8 to L9 and Fig. SM1(b) in Ref. [22] the ratio of the He survival probabilities from L1 to L9. Using these measured interaction probabilities the systematic error on the flux due to uncertainties of $\mathrm{He}$ inelastic cross sections was evaluated to be $1 \%$ below $100 \mathrm{GV}$. At higher rigidities, the small rigidity dependencies of the cross sections from the model were treated as an uncertainty and added in quadrature to the uncertainties from the measured interaction probabilities and the systematic error on the flux evaluated to be $2 \%$ at $3 \mathrm{TV}$.

An additional systematic error on the flux is due to differences in ${ }^{3} \mathrm{He}$ and ${ }^{4} \mathrm{He}$ interactions with the detector materials. This was assigned according to the uncertainty in the He isotopic composition. The error of $0.3 \%$ on the flux was obtained by varying the ratio of ${ }^{3} \mathrm{He} /{ }^{4} \mathrm{He}$ in the simulation from 0 to 0.2 [23]. The flux was then treated as containing only ${ }^{4} \mathrm{He}$.

The rigidity resolution function $\Delta(1 / R)$ for helium is similar to that of the protons shown in Fig. 1 of Ref. [8]. It has a pronounced Gaussian core characterized by width $\sigma$ and non-Gaussian tails more than $2.5 \sigma$ away from the center. The width of the core of the rigidity resolution function varies from $\sigma /(1 / R)=9 \%$ at $10 \mathrm{GV}$ to $14 \%$ at $100 \mathrm{GV}, 20 \%$ at $400 \mathrm{GV}$, and $64 \%$ at 2 TV. The nonGaussian tails of the rigidity resolution function amount to $3 \%$ at $10 \mathrm{GV}, 6 \%$ at $100 \mathrm{GV}, 11 \%$ at $400 \mathrm{GV}$, and $8 \%$ at $2 \mathrm{TV}$. The rigidity resolution function was obtained from the simulations and extensively verified with the data. We list four verifications. First, the differences of the coordinates measured in L3 or L5 to those obtained from the track fit using the measurements from L1, L2, L4, L6, L7, L8, and L9 were compared between data and simulation. This procedure directly measures the tracker coordinate accuracy of $\pm 7.5 \mu \mathrm{m}$ as shown Fig. SM1(c) in Ref. [22]. Similar results were obtained for the rest of the inner tracker layers. Second, the differences between the coordinates measured in L1 and L9 and those obtained from the track fit using the information from only the inner tracker were compared between data and simulation. Third, in order to validate the alignment of the external layers L1 and L9, the difference between the rigidities measured using the information from L1 to L8 and from L2 to L9 was compared between data and the simulation. Fourth, the RICH velocity resolution is $\Delta \beta / \beta=8.0 \times 10^{-4}$ [24]. The rigidity resolution function up to $20 \mathrm{GV}$, including nonGaussian tails, was obtained with data using the RICH velocity measurements only and compared with the rigidity resolution function from the simulation. The first three verifications provided the MDR and its uncertainty of $3.2 \pm 0.16 \mathrm{TV}$. The second and fourth provided the uncertainty of the amplitude of the non-Gaussian tails in the rigidity resolution function to be $10 \%$.

The systematic error on the He flux due to uncertainties in the rigidity resolution function was obtained by varying the width of the Gaussian core of the resolution function by $5 \%$ and the amplitude of the non-Gaussian tails by $10 \%$ over the entire rigidity range in the unfolding procedures and found to be less than $1 \%$ below $400 \mathrm{GV}$ and $3.5 \%$ at $3 \mathrm{TV}$. The small differences in the results from the two unfolding procedures $(0.25 \%$, at $2 \mathrm{GV},<0.1 \%$ between 2.5 and $200 \mathrm{GV}$, and $0.4 \%$ at $3 \mathrm{TV}$ ) were also accounted for as a systematic error. We have checked the sensitivity of the results to the binning by increasing the bin width by factors of 2 and 4 as well as reducing the bin width by factors of 2 and 4 . The resulting uncertainty is well within the assigned systematic errors.

There are two contributions to the systematic uncertainty on the rigidity scale, discussed in detail in Ref. [8]. The first is due to residual tracker misalignment. This error was estimated by comparing the $E / p$ ratio for electrons and positrons, where $E$ is the energy measured with the ECAL and $p$ is the momentum measured with the tracker. It was found to be $1 / 26 \mathrm{TV}^{-1}$, limited by the current high energy positron statistics. The second systematic error on the rigidity scale arises from the magnetic field map measurement and its temperature corrections. The error on the $\mathrm{He}$ flux due to uncertainty on the rigidity scale is below $0.6 \%$ up to $100 \mathrm{GV}$ and reaches $6 \%$ at $3 \mathrm{TV}$.

To ensure that the treatment of systematic errors is correct, several additional, independent verifications were performed. Figure SM2 in Ref. [22] shows examples of the stability of the measured flux for different conditions (presented as the ratio to the average flux) along with the corresponding systematic errors. Figure SM2(a) in Ref. [22] shows the independence of the integral flux above $30 \mathrm{GV}$, i.e., above the maximum geomagnetic cutoff, on the angle $\theta$ between the incoming helium direction and the AMS central axis; this verifies the systematic error on the acceptance. Figure SM2(b) in Ref. [22] shows the monthly integral flux above $45 \mathrm{GV}$ is constant within the systematic error of $1 \%$. This verifies that the detector 
performance is stable over time and that the flux above $45 \mathrm{GV}$ shows no observable effect from solar modulation fluctuations. Figure SM2(c) in Ref. [22] shows that the flux obtained using the rigidity measured by only the inner tracker is in good agreement with the flux measured using the full lever arm. The flux ratio uses the two different event samples corresponding to the inner tracker acceptance and to the L1 to L9 acceptance used for the results in this Letter. This verifies the systematic errors from the acceptance, the unfolding procedure, and the rigidity resolution function for two extreme and important cases. First, at the MDR of the inner tracker, $0.55 \mathrm{TV}$, where the unfolding effects and resolution functions of the inner tracker and the full lever arm are very different. Second, at low rigidities (2 to $10 \mathrm{GV}$ ) where the unfolding effects and the tails in the resolution functions of the inner tracker and full lever arm are also very different due to multiple and nuclear scattering. Figure SM2(d) in Ref. [22] shows the good agreement between the flux obtained using the rigidity measured by tracker L1 to L8, MDR 1.4 TV, and the full lever arm, MDR 3.2 TV, again using different event samples, thus verifying the systematic errors on the rigidity resolution function over the extended rigidity range.

Most importantly, several independent analyses were performed on the same data sample by different study groups. The results of those analyses are consistent with this Letter.

Results.-The measured He flux $\Phi$ including statistical errors and systematic errors is tabulated in Ref. [22], Table I, as a function of the rigidity at the top of the AMS detector. The contributions to the systematic errors come from (i) the trigger, (ii) the geomagnetic cutoff, the acceptance, and background contamination, (iii) the rigidity resolution function and unfolding which take into account the small differences between the two unfolding procedures described above, and (iv) the absolute rigidity scale. The contribution of individual sources to the systematic error are added in quadrature to arrive at the total systematic uncertainty. The Monte Carlo event samples have sufficient statistics such that they do not contribute to the errors. Figure 1(a) shows the flux as a function of rigidity with the total errors, the sum in quadrature of statistical and systematic errors [25]. In this and the subsequent figures, the points are placed along the abscissa at $\tilde{R}$ calculated for a flux $\propto R^{-2.7}$ [26]. Figure 1(b) shows the AMS flux as a function of kinetic energy per nucleon $E_{K}$ together with the most recent results (i.e., from experiments after the year 2000).

A power law with a constant spectral index $\gamma$,

$$
\Phi=C R^{\gamma},
$$

where $R$ is in $\mathrm{GV}$ and $C$ is a normalization factor, does not fit the flux reported in this work [22] and shown in Fig. 1(a) at the $99.9 \%$ C.L. for $R>45 \mathrm{GV}$. Applying solar modulation in the force field approximation [27] also does not fit the data at the $99.9 \%$ C.L. for $R>45 \mathrm{GV}$. We therefore
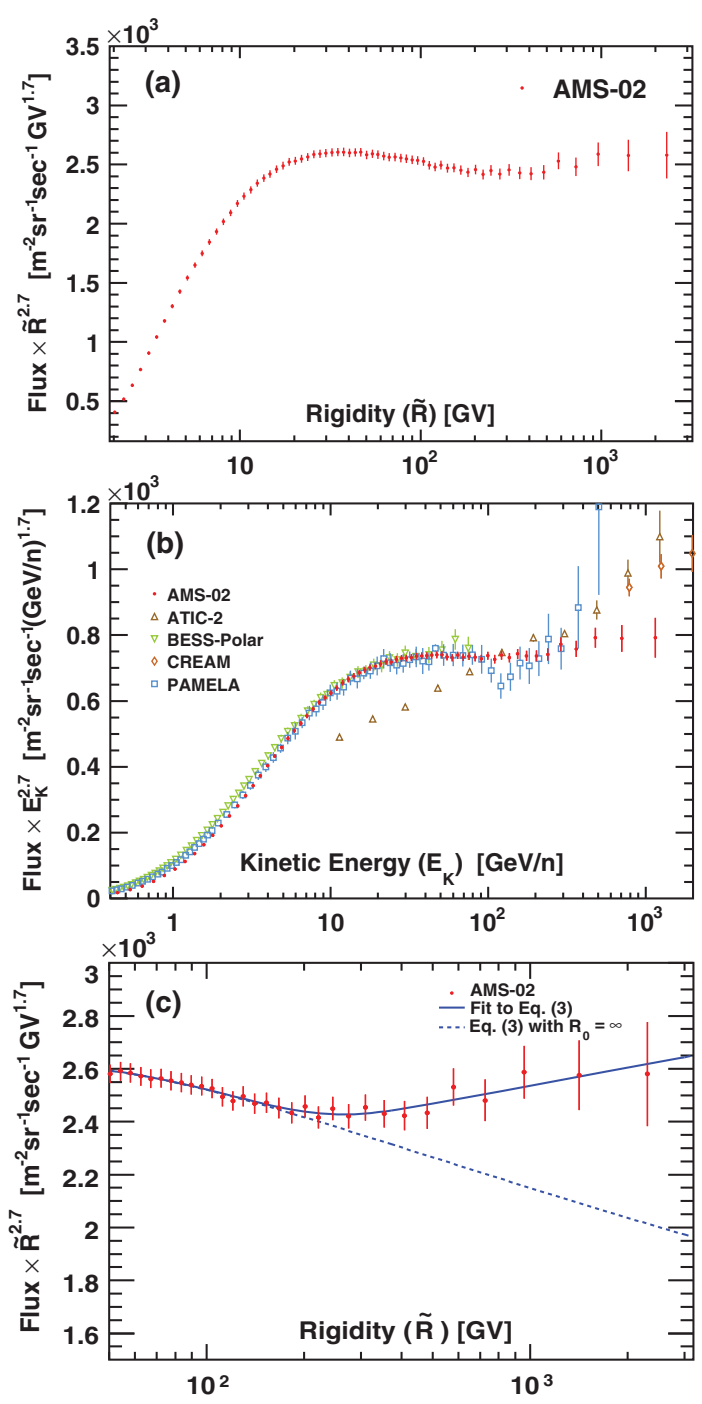

FIG. 1 (color). (a) The AMS helium flux [22] multiplied by $\tilde{R}^{2.7}$ with its total error as a function of rigidity. (b) The flux as a function of kinetic energy per nucleon $E_{K}$ multiplied by $E_{K}^{2.7}$ compared with measurements since the year 2000 [3-6]. For the AMS results $E_{K} \equiv\left(\sqrt{4 \tilde{R}^{2}+M^{2}}-M\right) / 4$ where $M$ is the ${ }^{4} \mathrm{He}$ mass as the AMS flux was treated as containing only ${ }^{4} \mathrm{He}$. (c) Fit of Eq. (3) to the AMS helium flux. For illustration, the dashed curve uses the same fit values but with $R_{0}$ set to infinity.

fit the flux with a double power law function [8]

$$
\Phi=C\left(\frac{R}{45 \mathrm{GV}}\right)^{\gamma}\left[1+\left(\frac{R}{R_{0}}\right)^{\Delta \gamma / s}\right]^{s},
$$

where $s$ quantifies the smoothness of the transition of the spectral index from $\gamma$ for rigidities below the characteristic transition rigidity $R_{0}$ to $\gamma+\Delta \gamma$ for rigidities above $R_{0}$. Fitting over the range $45 \mathrm{GV}$ to $3 \mathrm{TV}$ yields a $\chi^{2} /$ d.f. $=$ $25 / 27$ with $C=0.0948 \pm 0.0002$ (fit) \pm 0.0010 (sys) \pm 0.0006 (sol) $\mathrm{m}^{-2} \mathrm{sr}^{-1} \mathrm{sec}^{-1} \mathrm{GV}^{-1}, \gamma=-2.780 \pm 0.005$ (fit) \pm 0.001 (sys) \pm 0.004 (sol), $\Delta \gamma=0.119_{-0.010}^{+0.013}$ (fit) ${ }_{-0.028}^{+0.033}$ (sys) \pm $0.004(\mathrm{sol}), \quad s=0.027_{-0.010}^{+0.014}(\mathrm{fit})_{-0.013}^{+0.017}$ (sys) \pm 0.002 ( $\left.\mathrm{sol}\right)$, 
and $R_{0}=245_{-31}^{+35}(\text { fit })_{-30}^{+33}$ (sys) \pm 3 (sol) GV. The first error quoted (fit) takes into account the statistical and uncorrelated systematic errors from the flux reported in this work [22]. The second (sys) is the error from the remaining systematic errors, namely, from the rigidity resolution function and unfolding, and from the absolute rigidity scale, with the bin-to-bin correlations properly accounted. The third (sol) is the uncertainty due to the variation of the solar potential $\phi=0.50$ to $0.62 \mathrm{GV}$ [28]. The fit confirms that above $45 \mathrm{GV}$ the flux is incompatible with a single spectral index at the $99.9 \%$ C.L. The fit is shown in Fig. 1(c). For illustration, the fit results with $R_{0}$ set to infinity are also shown.

We observe that our measured positron fraction [29] and our measurements of the fluxes for helium, Fig. 1(c), and protons, [8] Fig. 4(a), all change their behavior at about the same rigidity. This can also be seen from the similarity of the $R_{0}$ for helium and proton fluxes, $R_{0}$ (protons) $=$ $336_{-44}^{+68}$ (fit) ${ }_{-28}^{+66}$ (sys) \pm 1 (sol) GV (where the errors have the same meanings), and the maximum of the positron fraction $E_{0}=275 \pm 32 \mathrm{GeV}$.

To obtain the detailed variation of $\gamma$ with rigidity in a model independent way, the spectral index is calculated from

$$
\gamma=d[\log (\Phi)] / d[\log (R)]
$$

over nonoverlapping rigidity intervals above $8.48 \mathrm{GV}$, see Ref. [22], with a variable width to have sufficient sensitivity to determine $\gamma$. The results are presented in Fig. 2(a) together with our measured proton spectral index [8]. As seen, the magnitude of the helium spectral index is different from that of the proton spectral index but the rigidity dependence is similar for helium and protons. In particular, both spectral indices progressively harden with rigidity above $100 \mathrm{GV}$.

To examine the difference between the rigidity dependence of the proton and helium fluxes, the ratio of the proton flux to the helium flux, or $p / \mathrm{He}$ ratio, was computed using data published in Ref. [8] and those tabulated in Ref. [22], Table I in the range where they overlap, from $1.9 \mathrm{GV}$ to $1.8 \mathrm{TV}$. This $p / \mathrm{He}$ ratio, including the statistical and systematic errors of the proton flux [8] and the helium flux, is tabulated in Ref. [22], Table II. The statistical errors are the sum in quadrature of the relative statistical errors of the proton and helium fluxes multiplied by the $p / \mathrm{He}$ ratio. The systematic errors from the trigger and acceptance are likewise added in quadrature. The correlations in the systematic errors from the unfolding and the absolute rigidity scale between the proton and helium fluxes have been accounted for in calculating the corresponding systematic errors of the $p / \mathrm{He}$ ratio. The contribution of individual sources to the systematic error are added in quadrature to arrive at the total systematic uncertainty. Figure 2(b) shows the AMS measurement with total errors,
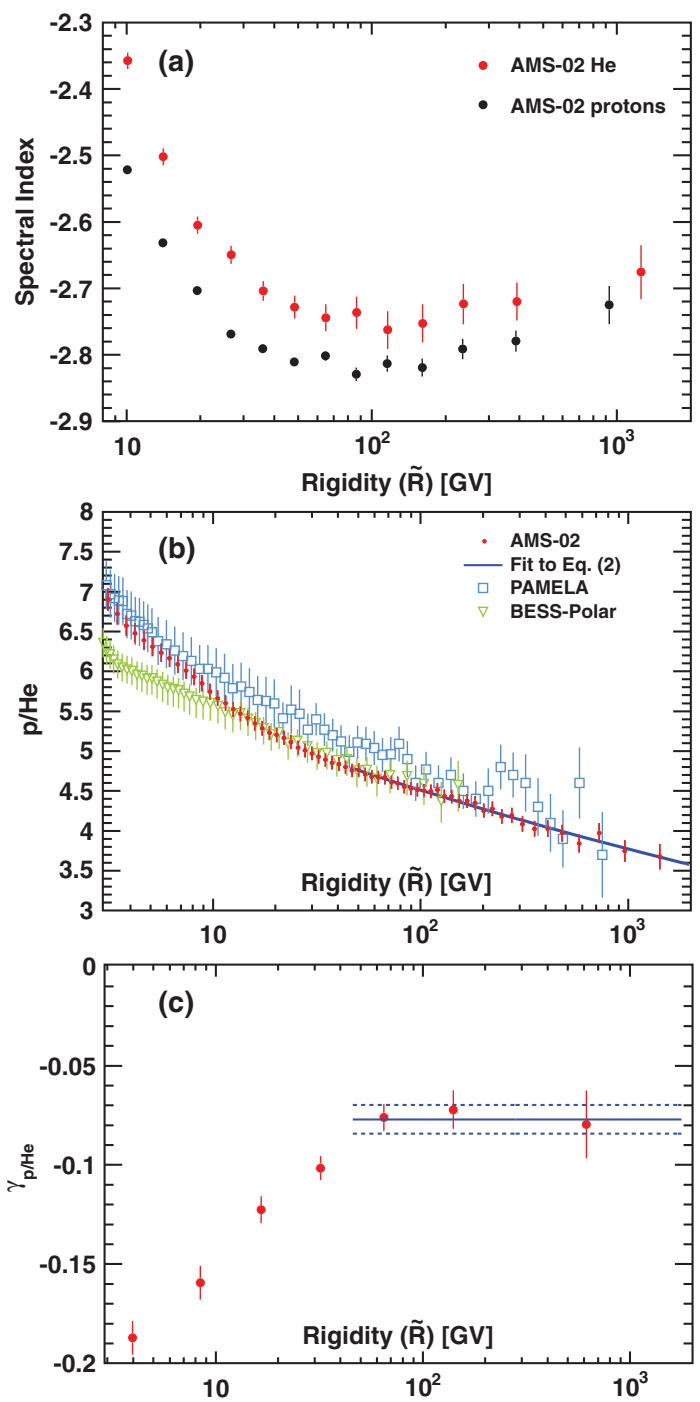

FIG. 2 (color). (a) The dependence of the helium and proton [8] spectral indices on rigidity. (b) The $p / \mathrm{He}$ ratio as a function of rigidity compared with recent measurements $[4,6]$. The solid blue curve indicates the fit of a single power law, Eq. (2), to the AMS data. As seen, above $45 \mathrm{GV}$ the ratio is well described by a single power law. (c) The rigidity dependence of the $p / \mathrm{He}$ spectral index $\gamma_{p / \mathrm{He}}$ as measured by AMS. As seen, $\gamma_{p / \mathrm{He}}$ increases up to about $45 \mathrm{GV}$. Above $45 \mathrm{GV}$ it becomes constant at $\gamma_{p / \mathrm{He}}=$ $-0.077 \pm 0.002$ (fit) \pm 0.007 (sys) as indicated by the solid blue line. The dashed blue lines are the total error (the sum in quadrature of the fit and systematic errors).

the quadratic sum of statistical and systematic errors, together with other recent measurements.

Above $45 \mathrm{GV}$ the $p / \mathrm{He}$ ratio measured by AMS is well fit with a single power law, Eq. (2), with a $\chi^{2} /$ d.f. $=22 / 29$ and a spectral index of $\gamma_{p / \mathrm{He}}=-0.077 \pm 0.002$ (fit) \pm 0.007 (sys). The first error quoted (fit) takes into account the statistical and uncorrelated systematic errors from the flux ratio reported in this work [22], Table II. The second (sys) is the error from the remaining systematic errors, namely, from the rigidity resolution function, unfolding and 
$p$ and He inelastic cross sections, with proper accounting of the bin-to-bin correlations.

Figure 2(c) shows the detailed variation of $\gamma_{p / \mathrm{He}}$ with rigidity over nonoverlapping rigidity intervals. As seen, the spectral index of the ratio increases with rigidity up to about $45 \mathrm{GV}$. Above $45 \mathrm{GV}$ it becomes constant.

In conclusion, precise knowledge of the helium flux is important in understanding the origin, acceleration, and propagation of cosmic rays. Previous measurements of the helium flux in cosmic rays have reported different variations of the flux with energy (or rigidity) and this has generated many theoretical models. Our precise measurement of the He flux from $1.9 \mathrm{GV}$ to $3 \mathrm{TV}$ is based on 50 million events with detailed studies of the systematic errors. The flux deviates from a single power law and the spectral index progressively hardens at rigidities larger than $100 \mathrm{GV}$. The magnitude of the helium spectral index is different from that of the proton spectral index, but the rigidity dependence is similar for helium and protons. The proton to helium flux ratio spectral index increases with rigidity up to about $45 \mathrm{GV}$ and becomes constant for higher rigidities; i.e., the proton to helium flux ratio is well described by a single power law above $45 \mathrm{GV}$.

We thank former NASA Administrator Daniel S. Goldin for his dedication to the legacy of the ISS as a scientific laboratory and his decision for NASA to fly the AMS as a DOE payload. We also acknowledge the continuous support of the NASA leadership including Charles Bolden, William H. Gerstenmaier, and Michael T. Suffredini and of the JSC and MSFC flight control teams which has allowed the AMS to operate optimally on the ISS for over four years. We are grateful for the support of Jim Siegrist and Michael Salamon of the DOE. We also acknowledge the continuous support from MIT and its School of Science, Michael Sipser, Marc Kastner, Ernest Moniz, and Richard Milner. We acknowledge the important contribution of P. Dennett. Research supported by São Paulo Research Foundation (FAPESP) Grants No. 2014/19149-7 and No. 2014/50747-8, Brazil; CAS, NSFC, MOST, National Laboratory for Aeronautics and Astronautics, the provincial governments of Shandong, Jiangsu, Guangdong, and the China Scholarship Council, China; the Finnish Funding Agency for Innovation (Tekes) Grants No. 40361/01 and No. 40518/03 and the Academy of Finland Grant No. 258963, Finland; CNRS, IN2P3, CNES, Enigmass, and the ANR, France; J. Trümper, J. D. Woerner, DLR, and JARA-HPC under Project No. JARA0052, Germany; INFN and ASI under ASI-INFN Agreements No. 2013-002-R.0 and No. 2014-037-R.0, Italy; Grants No. NRF-20090080142 and No. NRF-2012-010226 at CHEP, Kyungpook National University and No. NRF-2013004883 at Ewha Womans University, Korea; the Consejo Nacional de Ciencia y Tecnología at UNAM, Mexico; CIEMAT, IAC, SEIDI MINECO, CDTI, and Centro Nacional de Física de Partículas, Astropartículas y
Nuclear, Spain; the Swiss National Science Foundation (SNSF), federal and cantonal authorities, Switzerland; Academia Sinica and the Ministry of Science and Technology (MOST) under Grants No. 103-2682-M-008002 and No. 103-2112-M-001-036, former President of Academia Sinica Yuan-Tseh Lee, and former Ministers of MOST Maw-Kuen Wu and Luo-Chuan Lee, Taiwan; and the Turkish Atomic Energy Authority at METU, Turkey. We gratefully acknowledge the strong support from CERN, including Rolf-Dieter Heuer, and from the European Space Agency. We are grateful for important discussions with Barry Barish, Jonathan Ellis, Jonathan Feng, Igor Moskalenko, Steve Olsen, George Smoot, Michael Turner, Steven Weinberg, Frank Wilczek, and Arnold Wolfendale.

\footnotetext{
*Deceased.

${ }^{a}$ Also at ASI, I-00133 Roma, Italy.

${ }^{\mathrm{b}}$ Present address: Departamento de Ciencias, Pontifica Universidad Católica del Perú (PUCP), Lima 32, Peru.

${ }^{\mathrm{c}}$ Present address: CP3, Université catholique de Louvain, Belgium.

${ }^{\mathrm{d}}$ Also at ASI Science Data Center (ASDC), I-00133 Roma, Italy.

'Also at Xi' an Jiaotong University (XJTU), Xi' an 710049, China.

${ }^{\mathrm{f}}$ Also at Sun Yat-Sen University (SYSU), Guangzhou 510275, China.

${ }^{\mathrm{g}}$ Present address: SRON, Utrecht, Netherlands.

${ }^{\mathrm{h}}$ Present address: CERN, CH-1211 Geneva 23, Switzerland. ${ }^{i}$ Also at Shandong University (SDU), Jinan, Shandong 250100, China.

${ }^{\mathrm{j} A l s o}$ at Harbin Institute of Technology (HIT), Harbin 150001, China.

${ }^{\mathrm{k}}$ Also at Huazhong University of Science and Technology (HUST), Wuhan 430074, China.

${ }^{1}$ Also at University of Science and Technology of China (USTC), Hefei 230026, China.

${ }^{\mathrm{m}}$ Also at Università di Siena I-53100 Siena, Italy.

${ }^{\mathrm{n}}$ Also at Laboratoire d'Annecy-le-Vieux de Physique Théorique (LAPTh), CNRS and Université Savoie Mont Blanc, F-74941 Annecy-le-Vieux, France.

${ }^{\circ}$ Also at Nankai University, Tianjin 300071, China.

${ }^{\mathrm{p}}$ Also at Beijing Normal University (BNU), Beijing 100875, China.

${ }^{\mathrm{q}}$ Also at Southeast University (SEU), Nanjing 210096, China.
}

[1] I. A. Grenier, J. H. Black, and A. W. Strong, Annu. Rev. Astron. Astrophys. 53, 199 (2015); P. Blasi, Astron. Astrophys. Rev. 21, 70 (2013); A. W. Strong, I. V. Moskalenko, and V.S. Ptuskin, Annu. Rev. Nucl. Part. Sci. 57, 285 (2007).

[2] V. A. Derbina et al., Astrophys. J. 628, L41 (2005); M. Boezio et al., Astropart. Phys. 19, 583 (2003); E. Diehl, D. Ellithorpe, D. Muller, and S. P. Swordy, Astropart. Phys. 18, 487 (2003); J. Alcaraz et al., Phys. Lett. B 494, 193 (2000); W. Menn et al., Astrophys. J. 533, 281 (2000); R. Bellotti et al., Phys. Rev. D 60, 052002 (1999); M. Boezio et al., Astrophys. J. 518, 457 (1999); K. Asakimori et al., 
Astrophys. J. 502, 278 (1998); I. P. Ivanenko et al., in Proceedings of the 23rd International Cosmic Ray Conference, Calgary (World Scientific, Singapore, 1993), p. 17.

[3] For the ATIC experiment, see A. D. Panov et al., Bull. Russ. Acad. Sci.: Phys. 73, 564 (2009); extracted from D. Maurin, F. Melot, and R. Taillet, Astron. Astrophys. 569, A32 (2014).

[4] For the BESS experiment, see K. Abe et al., arXiv:1506.01267 [Astrophys. J. (to be published)]; Y. Shikaze et al., Astropart. Phys. 28, 154 (2007); S. Haino et al., Phys. Lett. B 594, 35 (2004); T. Sanuki et al., Astrophys. J. 545, 1135 (2000).

[5] For the CREAM experiment, see Y.S. Yoon et al., Astrophys. J. 728, 122 (2011).

[6] For the PAMELA experiment, see O. Adriani et al., Science 332, 69 (2011).

[7] See, for example, A. D. Erlykin and A. W. Wolfendale, J. Phys. G 42, 075201 (2015); S. Thoudam and J. R. Hörandel, Astron. Astrophys. 567, A33 (2014); G. Bernard, T. Delahaye, Y.-Y. Keum, W. Liu, P. Salati, and R. Taillet, Astron. Astrophys. 555, A48 (2013); V. S. Ptuskin, V. Zirakashvili, and E. S. Seo, Astrophys. J. 763, 47 (2013); N. Tomassetti, Astrophys. J. Lett. 752, L13 (2012); P. Blasi, E. Amato, and P. D. Serpico, Phys. Rev. Lett. 109, 061101 (2012); A. E. Vladimirov, G. Jóhannesson, I. V. Moskalenko, and T. A. Porter, Astrophys. J. 752, 68 (2012); M. A. Malkov, P. H. Diamond, and R. Z. Sagdeev, Phys. Rev. Lett. 108, 081104 (2012); L. A. Fisk and G. Gloeckler, Astrophys. J. 744, 127 (2012); Y. Ohira and K. Ioka, Astrophys. J. Lett. 729, L13 (2011).

[8] M. Aguilar et al., Phys. Rev. Lett. 114, 171103 (2015).

[9] A. Kounine, Int. J. Mod. Phys. E 21, 1230005 (2012); S. Rosier-Lees, in Proceedings of Astroparticle Physics TEVPA/IDM, Amsterdam, 2014 (to be published); S. Ting, Nucl. Phys. B, Proc. Suppl. 243-244, 12 (2013); S.-C. Lee, in Proceedings of the 20th International Conference on Supersymmetry and Unification of Fundamental Interactions (SUSY 2012), Beijing, 2012 (unpublished); M. Aguilar, in Proceedings of the XL International Meeting on Fundamental Physics, Centro de Ciencias de Benasque Pedro Pascual, 2012 (unpublished); S. Schael, in Proceedings of the 10th Symposium on Sources and Detection of Dark Matter and Dark Energy in the Universe, Los Angeles, 2012 (unpublished); B. Bertucci, Proc. Sci., EPS-HEP (2011) 67; M. Incagli, AIP Conf. Proc. 1223, 43 (2010); R. Battiston, Nucl. Instrum. Methods Phys. Res., Sect. A 588, 227 (2008).

[10] K. Lübelsmeyer et al., Nucl. Instrum. Methods Phys. Res., Sect. A 654, 639 (2011).

[11] B. Alpat et al., Nucl. Instrum. Methods Phys. Res., Sect. A 613, 207 (2010).

[12] V. Bindi et al., Nucl. Instrum. Methods Phys. Res., Sect. A 743, 22 (2014), and references therein.

[13] J. Allison et al., IEEE Trans. Nucl. Sci. 53, 270 (2006); S. Agostinelli et al., Nucl. Instrum. Methods Phys. Res., Sect. A 506, 250 (2003).

[14] A. Boudard, J. Cugnon, J.-C. David, S. Leray, and D. Mancusi, Phys. Rev. C, 87, 014606 (2013); S. Leray, D. Mancusi, P. Kaitaniemi, J. C. David, A. Boudard, B. Braunn, and J. Cugnon, J. Phys. Conf. Ser. 420, 012065 (2013).

[15] J. Ranft, Phys. Rev. D 51, 64 (1995).

[16] V. G. Ableev et al., Acta Phys. Pol. B 16, 913 (1985).
[17] J. Alcaraz et al., Phys. Lett. B 484, 10 (2000).

[18] C. C. Finlay et al., Geophys. J. Int. 183, 1216 (2010). We have used data from IGRF-12 (2015), currently available at http://www.ngdc.noaa.gov/IAGA/vmod/igrf.html (unpublished).

[19] AMS Collaboration, Measurement of the Flux of Light Nuclei in Primary Cosmic Rays with the Alpha Magnetic Spectrometer on the International Space Station (to be published).

[20] A. Ingemersson et al., Nucl. Phys. A676, 3 (2000); A. Auce, R. F. Carlson, A. J. Cox, A. Ingemarsson, R. Johansson, P. U. Renberg, O. Sundberg, G. Tibell, and R. Zorro, Phys. Rev. C 50, 871 (1994); I. Tanihata et al., Phys. Lett. B 160, 380 (1985); V. D. Aksinenko et al., Nucl. Phys. A348, 518 (1980); E. O. Abdrahmanov et al., Z. Phys. C 5, 1 (1980); J. Jaros et al., Phys. Rev. C 18, 2273 (1978).

[21] AMS Collaboration, Measurement of Nuclear Interaction Cross Sections with the Alpha Magnetic Spectrometer on the International Space Station (to be published).

[22] See Supplemental Material at http://link.aps.org/ supplemental/10.1103/PhysRevLett.115.211101 for the tabulated helium flux and proton-helium flux ratio and figures on the survival probability of helium crossing the detector, the differences of the reconstructed inner tracker coordinates between data and simulation, and the independent verification of the assigned systematic errors.

[23] AMS Collaboration, Measurement of the He isotopic composition in Primary Cosmic Rays with the Alpha Magnetic Spectrometer on the International Space Station (to be published); O. Adriani et al., Astrophys. J. 770, 2 (2013); B. Coste, L. Derome, D. Maurin, and A. Putze, Astron. Astrophys. 539, A88 (2012); M. Aguilar et al., Astrophys. J. 736, 105 (2011); Z. D. Myers et al., in Proceedings of the 28th International Cosmic Ray Conference, Tsukuba, 2003, edited by T. Kajita, Y. Asaoka, A. Kawachi, M. Sasaki, and Y. Matsubara (Universal Academy Press, Tokyo, 2003), p. 1805; J. Z. Wang et al., Astrophys. J. 564, 244 (2002); S. P. Ahlen et al., Astrophys. J. 534, 757 (2000); O. Reimer et al., Astrophys. J. 496, 490 (1998); Y. Hatano, Y. Fukada, T. Saito, H. Oda, and T. Yanagita, Phys. Rev. D 52, 6219 (1995); J. J. Beatty et al., Astrophys. J. 413, 268 (1993); S. P. Jordan et al., Astrophys. J. 291, 207 (1985).

[24] F. Giovacchini, Nucl. Instrum. Methods Phys. Res., Sect. A 766, 57 (2014).

[25] A progress report on our helium analysis was presented at the 33rd International Cosmic Ray Conference (2013). At that time our understanding of the systematic errors did not allow an accurate determination of the behavior of the helium flux.

[26] G. D. Lafferty and T. R. Wyatt, Nucl. Instrum. Methods Phys. Res., Sect. A 355, 541 (1995). We have used Eq. (6) with $\tilde{R} \equiv x_{l w}$.

[27] L. J. Gleeson and W. I. Axford, Astrophys. J. 154, 1011 (1968).

[28] I. G. Usoskin, G. A. Bazilevskaya, and G. A. Kovaltsov, J. Geophys. Res. 116, A02104 (2011); K. G. McCracken and J. Beer, J. Geophys. Res. 112, A10101 (2007); I. G. Usoskin, K. Alanko-Huotari, G. A. Kovaltsov, and K. Mursula, J. Geophys. Res. 110, A12108 (2005). We have also used more recent data from: http://cosmicrays.oulu.fi/ phi/Phi_mon.txt.

[29] L. Accardo et al., Phys. Rev. Lett. 113, 121101 (2014). 